\title{
Pranlukast hydrate in the treatment of pediatric bronchial asthma
}

This article was published in the following Dove Press journal:

Pediatric Health, Medicine and Therapeutics

8 July 2013

Number of times this article has been viewed

\section{Shigemi Yoshihara}

Department of Pediatrics, Dokkyo Medical University, Tochigi, Japan
Correspondence: Shigemi Yoshihara Department of Pediatrics, Dokkyo Medical University, 880 Kitakobayashi, Mibu-machi, Shimotsuga-gun,

Tochigi 32I-0293, Japan

$\mathrm{Tel}+8 \mid 282872$ I 55

Fax +8I 28286752 I

Email shigemi@dokkyomed.ac.jp
Abstract: Pranlukast hydrate is a potent, selective, orally active, cysteinyl leukotriene antagonist that binds at the type 1 receptor. It is used as a 10\% dry syrup to treat asthma and allergic rhinitis in pediatric patients. In a 4-week, dose-finding study, a dose-dependent improvement in lung function was observed in pediatric patients with asthma at an optimal dose of 5.1-10 mg/kg/ day. In a comparative, randomized, double-blind, 4-week multicenter trial, pranlukast dry syrup $7 \mathrm{mg} / \mathrm{kg}$ /day achieved significantly better final overall improvement (71.4\%) versus oxatomide $1 \mathrm{mg} / \mathrm{kg} /$ day $(37.2 \%)$ in pediatric patients older than one year with asthma. In two 12-week, open-label trials and in a long-term open-label trial of treatment for up to 24 months, pranlukast dry syrup improved asthma control over baseline values. In a prospective post-marketing surveillance study and a long-term follow-up study, the safety and efficacy of pranlukast dry syrup was confirmed in infants younger than one year with bronchial asthma. In a 4-week, open-label trial, pranlukast dry syrup improved overall health-related quality of life and physical and emotional domain scores over baseline in pediatric patients with asthma. In a randomized, multicenter, double-blind, placebo-controlled, two-period crossover trial, pranlukast dry syrup significantly inhibited exercise-induced bronchospasm in children with asthma compared with placebo. In a modified Childhood Asthma Control Test, pranlukast dry syrup was significantly more effective in controlling asthma in patients younger than 4 years with the common cold. These findings show that pranlukast is useful and beneficial for treating pediatric patients with bronchial asthma.

Keywords: pranlukast, leukotriene receptor antagonist, pediatric asthma

\section{Introduction}

Global Initiative for Asthma guidelines define asthma as a chronic inflammatory disorder characterized by hyper-responsiveness and narrowing or obstruction of the airways in response to allergenic and other factors, including exercise and exposure to cold air, and limiting of usually reversible and variable widespread airflow. ${ }^{1}$ An early asthmatic response is characterized by rapid onset of bronchoconstriction lasting 1-3 hours after exposure to an allergen. A late asthmatic response, ie, a more protracted bronchoconstriction, is associated with bronchial hyper-responsiveness and an influx of eosinophils into the bronchi occurring several hours after an early asthmatic response, which may last up to 12 hours and is linked with cysteinyl leukotriene activity. ${ }^{2-4}$

Cobra venom studies in an animal model over 70 years ago identified a chemical mediator causing sustained contraction of smooth muscle in the airways. ${ }^{5}$ The action of this compound appeared to last longer than that of histamine, and researchers suggested that both mediators may have a role in the management of asthma. ${ }^{6}$ The mediator, 
termed a slow-reacting substance of anaphylaxis, was later identified in lung tissue from asthmatic patients, ${ }^{7}$ and is now known to consist of the inflammatory mediators leukotriene (LT)C4, LTD4, and LTE4, ie, cysteinyl leukotrienes. ${ }^{8}$ Leukotrienes are formed when arachidonic acid, a component of the multicellular phospholipid bilayer, is oxidatively metabolized. Cysteinyl leukotrienes act physiologically in humans by binding to and activating the cysteinyl leukotriene 1 (CysLTl) and 2 (CysLT2) receptor subtypes. ${ }^{13-16}$ In humans, LTC4, LTD4, and LTE4 also bind to CysLTl receptors and are recognized as important mediators in bronchial asthma. ${ }^{17-19}$ CysLT2 receptors mediate the constriction of pulmonary vascular smooth muscle in humans, although the mechanism involved is not as well understood as that for CysLTl receptors. ${ }^{16,20-23}$ Recognition of the role of leukotrienes in the pathogenesis of asthma subsequently led to the development of leukotriene receptor antagonists.

Pranlukast hydrate, a potent selective competitive antagonist at the CysLTl receptor, inhibits the activation of endogenous and exogenous LTC4, LTD4, and LTE4 receptors. ${ }^{24}$ Pranlukast, the first leukotriene receptor antagonist to be developed, was introduced in Japan in 1995 to treat asthma, and is also now indicated in the treatment of allergic rhinitis. Pranlukast is approved for use as a capsule formulation in adults and as a dry syrup formulation in children (Figure 1).

The recommended dose of pranlukast is $450 \mathrm{mg}$ /day in adult patients and $7 \mathrm{mg} / \mathrm{kg} /$ day (which can be increased to a maximum of $10 \mathrm{mg} / \mathrm{kg} /$ day provided that the total daily dose does not exceed $450 \mathrm{mg}$ ) in pediatric patients, which should be divided into two equal doses administered morning and evening. Because food affects the bioavailability of pranlukast, the drug needs to be administered after breakfast and dinner. Pranlukast dry syrup is a mixture containing $10 \%$ pranlukast that requires addition of water when dispensing. The poor chemical stability of the drug requires an aqueous vehicle to avoid problems of physical insta-

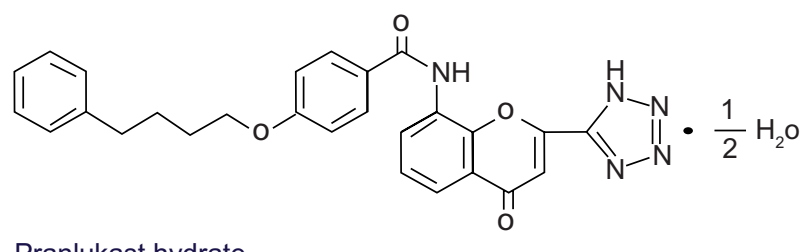

Pranlukast hydrate

4-Oxo-8-[4-(4-phenylbutoxy) benzoylamino]--2(tetrazol-y)-4H-1 -benzopyran hemihydrate

$\mathrm{C}_{27} \mathrm{H}_{23} \mathrm{~N}_{5} \mathrm{O}_{4} \cdot 1 / 2 \mathrm{H}_{2} \mathrm{O}$

490.51

Figure I Structural formula of pranlukast hydrate. bility. This paper reviews the efficacy and safety of pranlukast dry syrup in treating bronchial asthma in pediatric patients.

\section{Dose-finding in an open-label multicenter trial}

A dose-finding study was performed to establish the efficacy and safety of pranlukast dry syrup and to determine an optimal twice-daily dose in 134 pediatric patients with bronchial asthma. ${ }^{25}$ The efficacy of pranlukast dry syrup was evaluated by the patient, a parent, or a caregiver, for asthma symptoms (including asthma attacks, cough, daily life, sleep), lung function (peak morning and evening expiratory flow), use of additional drugs, and an asthma diary. Final overall improvement showed significant dose-dependency $(P<0.05)$ for three dose groups, at $46.0 \%$ for $2.6-5.0 \mathrm{mg} / \mathrm{kg} /$ day (group L), $73.8 \%$ for $5.1-10.0 \mathrm{mg} / \mathrm{kg} /$ day (group $\mathrm{M}$ ), and $66.7 \%$ for $10.1-15.0 \mathrm{mg} / \mathrm{kg} /$ day (group H). Usefulness in the three dose groups was also significantly dose-dependent $(P<0.05$, Cochrane-Armitage test) at $45.1 \%, 73.8 \%$, and $64.5 \%$, respectively. Final overall improvement was analyzed for patient groups based on dose increments of $2 \mathrm{mg} / \mathrm{kg} /$ day, revealing that improvement ranged from $62.5 \%$ to $77.8 \%$ at daily doses of $6.1 \mathrm{mg} / \mathrm{kg}$ or higher. Adverse reactions were reported in $3.8 \%, 2.2 \%$, and $8.6 \%$ of cases, respectively, and were mainly gastrointestinal in nature. Both final overall improvement and usefulness were dose-dependent in groups L and M. In group H, a higher rate of adverse reactions was observed, but this did not affect final overall improvement or usefulness. The frequency of asthma attacks (minor and all attacks) and cough decreased in each group, with a greater decrease observed in groups $\mathrm{M}$ and $\mathrm{H}$ compared with group L, but no significant intergroup differences were seen. In addition, morning and evening peak expiratory flows were significantly increased in each group, with a larger increase observed in groups $\mathrm{M}$ and $\mathrm{H}$ compared with group L, but again there were no significant differences between the groups. These results demonstrated clearly that pranlukast dry syrup could be beneficial in treating bronchial asthma in pediatric patients and that the optimal dose range is $5.1-10.0 \mathrm{mg} / \mathrm{kg} / \mathrm{day}$.

\section{Multicenter double-blind clinical comparison with oxatomide dry syrup}

The usefulness of pranlukast was compared with that of oxatomide dry syrup in a multicenter, randomized, doubleblind, parallel-group study in pediatric patients with bronchial asthma. ${ }^{26}$ The efficacy of pranlukast dry syrup was evaluated by the patient, a parent, or a caregiver, for asthma 
symptoms (asthma attacks, cough, daily life, sleep), lung function (morning and evening peak expiratory flow), use of additional drugs, and an asthma diary. Following a 2-week run-in period, 214 patients were administered $7 \mathrm{mg} / \mathrm{kg} / \mathrm{day}$ of pranlukast dry syrup (group O) or $1 \mathrm{mg} / \mathrm{kg} /$ day of oxatomide dry syrup (group K) for 4 weeks. Per protocol analysis patients numbered 99 in group $\mathrm{O}$ and 103 in group $\mathrm{K}$.

With regard to final overall improvement, efficacy differed significantly, being $71.4 \%$ in group $\mathrm{O}$ and $37.2 \%$ in group $\mathrm{K}$ ( $P=0.0001$, chi-square test). The $90 \%$ confidence limit for the difference in efficacy between the groups was found to be $+22.9 \%$ in group $\mathrm{O}$ and $+45.5 \%$ in group $\mathrm{K}$. Intent-to-treat analysis of final overall improvement gave the same results as those for the per protocol analysis. Group O showed a significant decrease in mild to moderate attacks and cough. With regard to concomitant antiasthma medication, use of inhaled bronchodilators and oral $\beta$-stimulants decreased significantly compared with baseline values. Group $\mathrm{K}$ also showed a significant decrease in mild attacks and cough, but no significant decrease in use of concomitant medication. Differences in reduction of inhaled bronchodilators and use of oral $\beta$-stimulants were significant between the groups. The percent predicted peak expiratory flow rate during 4 weeks of treatment in group $\mathrm{O}$ increased significantly in the morning and at bed time compared with baseline values, but no significant change was seen in group $\mathrm{K}$. There were no significant differences of the percent predicted peak expiratory flow rate between the two groups. Nine patients (9.4\%) in group $\mathrm{O}$ and 11 (11.1\%) in group K reported adverse events. Rating of usefulness differed significantly between the groups ( $P=0.0001$, chi-square test), being $67.4 \%$ in group $\mathrm{O}$ and $31.9 \%$ in group $\mathrm{K}$. The $90 \%$ confidence limit for the difference in usefulness between the groups was $+24.1 \%$ in group $\mathrm{O}$ and $+46.9 \%$ in group $\mathrm{K}$.

Intent-to-treat analysis of rating of usefulness yielded the same results as those in the per protocol analysis. Only pranlukast dry syrup showed significantly more efficacy in terms of reducing use of bronchodilators, and a decrease in predicted peak expiratory flow compared with oxatomide dry syrup. These efficacy results indicate significant differences in both final overall improvement and usefulness between the two dry syrup formulations. Based on the findings of this study, it can be concluded that pranlukast dry syrup shows efficacy equivalent to or exceeding that of oxatomide dry syrup and is a useful antiasthmatic drug for the treatment of pediatric patients with bronchial asthma (Table 1).

\section{Long-term use}

The efficacy and safety of pranlukast dry syrup in longterm use were evaluated in pediatric patients with bronchial asthma. ${ }^{27}$ Efficacy was evaluated by the patient, a parent, or caregiver, for asthma symptoms (asthma attacks, cough, daily life, sleep), lung function (morning and evening peak expiratory flow), use of concomitant drugs, and an asthma diary. Forty-one patients were analyzed (17 with atopic, 21 with mixed, and three with infectious etiology). The overall improvement rate was $52.5 \%$ at week $2,72.5 \%$ at week 4 , $72.5 \%$ at week $6,79.5 \%$ at week $8,68.3 \%$ at week 10 , and $82.9 \%$ at week 12 . The final overall improvement rate was $78.0 \%$, the overall safety rate was $95.1 \%$, and rate for evaluation of usefulness was $78.0 \%$. Stratified analysis of

Table I Efficacy of oral pranlukast dry syrup compared with that of oxatomide in the treatment of pediatric asthma

\begin{tabular}{|c|c|c|c|c|c|c|}
\hline & \multicolumn{3}{|c|}{ Oxatomide (103) } & \multicolumn{3}{|c|}{ Pranlukast (99) } \\
\hline & Baseline & $\begin{array}{l}\text { Weeks I-2 (change } \\
\text { from baseline) }\end{array}$ & $\begin{array}{l}\text { Weeks 3-4 (change } \\
\text { from baseline) }\end{array}$ & Baseline & $\begin{array}{l}\text { Weeks I-2 (change } \\
\text { from baseline) }\end{array}$ & $\begin{array}{l}\text { Weeks 3-4 (change } \\
\text { from baseline) }\end{array}$ \\
\hline \multicolumn{7}{|c|}{ No of occurrences (no/2 wk) } \\
\hline Mild attack & 4.88 & $-2.1^{* * *}$ & $-2.82^{* * *}$ & 5.21 & $-2.43^{\text {***** }}$ & $-3.09^{* * * *}$ \\
\hline Moderate atttack & 1.09 & -0.4 & -0.55 & 1.87 & $-1.32^{*}$ & $-1.54^{* *, a}$ \\
\hline Cough & 7.39 & $-2.02^{* *}$ & $-2.57^{* *}$ & 7.58 & $-2.17^{k+o k}$ & $-3.36^{\text {*** }}$ \\
\hline \multicolumn{7}{|c|}{ Use of bronchodilator (medication/2 wk) } \\
\hline Inhaled & 4.14 & -0.41 & -0.56 & 6.1 & $-1.99^{* *, a}$ & $-2.58^{* *, a}$ \\
\hline Oral $\beta$-agonists & 11.72 & -0.53 & -0.57 & $\mid 1.81$ & $-2.1^{* *, a}$ & $-3.21^{* * *, a}$ \\
\hline \multicolumn{7}{|l|}{ PEFR (L/ml) } \\
\hline am & 208.72 & 6.92 & 16.23 & 207.17 & 20.17 & $19.73^{*}$ \\
\hline $\mathrm{pm}$ & 232.42 & 11.59 & 16.82 & 238.75 & $15.73^{*}$ & $15.47^{*}$ \\
\hline \multicolumn{7}{|l|}{ Predicted PEFR (\%) } \\
\hline $\mathrm{am}$ & 68.34 & 1.88 & 4.7I & 70.97 & $7.4^{*}$ & $6.76^{*}$ \\
\hline $\mathrm{pm}$ & 70.85 & 3.48 & 5.35 & 75.08 & $5.35^{*}$ & $5.07^{*}$ \\
\hline
\end{tabular}

Notes: ${ }^{*} P<0.05, * * P<0.00 I,{ }^{*} * * P<0.000 \mathrm{I}$, vs baseline (paired $t$-test); ${ }^{\mathrm{a}}<<0.05$, vs oxatomide (t-test).

Abbreviation: PEFR = peak expiratory flow rate. 
the final overall improvement rate was $86.4 \%$ in a subgroup with moderate attacks and $66.7 \%$ in a subgroup with severe attacks. The incidence of attacks and use of steroids and bronchodilators had decreased significantly and rapidly by week 4, and was maintained up to week 12 . Adverse events were noted in two cases. Pranlukast dry syrup showed efficacy from week 2 onwards which was maintained between weeks 4 and 12. Efficacy in reducing attacks and need for concomitant medication continued between weeks 4 and 12. Therefore, pranlukast dry syrup had a sustained pharmacological action and therapeutic effect in pediatric patients with bronchial asthma over a long period. No safety problems were observed, making pranlukast a useful drug in the clinical setting.

\section{Long-term use for $\mathbf{2}$ weeks}

The efficacy and safety of pranlukast dry syrup in longterm use was evaluated in children with asthma. ${ }^{28}$ The efficacy of pranlukast dry syrup was evaluated by the patient, a parent, or a caregiver for asthma symptoms (attacks, cough, daily life, sleep), lung function (morning and evening peak expiratory flow), use of additional drugs, and an asthma diary. Fifty patients (38 with atopic, eight with mixed, and four with infectious etiology) were included on the study, 45 of whom received the drug for 12 weeks. Overall improvement rates measured at 2-week intervals increased as follows: $42.9 \%$ at week $2,60.4 \%$ at week $4,56.3 \%$ at week $6,60.9 \%$ at week $8,63.8 \%$ at week 10 , and $71.7 \%$ at week 12 . The final overall improvement rate was $68.1 \%$, rate of overall assessment of the product as "safe" was $96.0 \%$, and the rating of usefulness was $66.0 \%$. Final overall improvement was $65.7 \%$ in atopic patients and $62.5 \%$ in patients with asthma of mixed etiology. Usefulness according to severity was rated as $76.0 \%$ for those with moderate attacks and $57.9 \%$ for those with severe attacks. The number of attacks had decreased significantly and rapidly by week 4 , with a consistent effect maintained up to week 12 . For subjects with asthma of mild or moderate severity, use of inhaled bronchodilators and antiallergic drugs decreased significantly and rapidly by week 2 . One adverse event of urticaria was reported. Pranlukast dry syrup demonstrated marked efficacy between weeks 4 and 12, regardless of the etiology of asthma and degree of severity. Efficacy in terms of reducing use of inhaled bronchodilators and antiallergic drugs was demonstrated from week 2 onwards. Pranlukast dry syrup thus maintained its pharmacological action and therapeutic effect for a long period in children with asthma.

\section{Extended use for up to $\mathbf{3 6}$ months}

The efficacy of pranlukast dry syrup has been evaluated for up to 36 months in pediatric patients with mild to severe asthma (mean duration 13 months; data available for 24 months) in the largest open-label trial to date, which included 77 pediatric patients aged $1-15$ years. ${ }^{29}$ In this study, treatment with other antiallergy medication (ie, ketotifen, epinastine, pemirolast, azelastine, oxatomide, or suplatast tosilate) was switched to pranlukast dry syrup. The efficacy of pranlukast dry syrup was evaluated by the patient, a parent, or a caregiver, for asthma symptoms (asthma attacks, cough, daily life, sleep), lung function (morning and evening peak expiratory flow), use of additional drugs, and an asthma diary.

Treatment with pranlukast dry syrup for up to 24 months improved asthma control compared with baseline values. Patients treated with pranlukast dry syrup at a dose of $7 \mathrm{mg} / \mathrm{kg}$ /day had significantly fewer mild or moderate asthma attacks than at baseline at most assessment points up to 24 months $(P<0.05)$. This improvement was evident regardless of whether patients had $(n=33)$ or had not $(n=44)$ previously received antiallergy medication. Compared with baseline, pranlukast dry syrup for 12 months significantly improved asthma symptoms and inhaled $\beta 2$-agonist scores, and decreased the number of episodes of hospitalization or intravenous aminophylline treatment needed during emergency clinic visits $(P<0.05)$. Pranlukast dry syrup thus maintained a pharmacological effect and reduced the number of emergency clinic visits and hospitalizations over a longer period in children with asthma, showing a therapeutic effect independent of treatment with antiallergy medication.

\section{Prospective study in children aged younger than one year}

A post-marketing surveillance study investigated the safety and efficacy of pranlukast dry syrup in actual clinical settings involving pediatric patients aged younger than one year with bronchial asthma. ${ }^{30}$ Diagnosis of asthma in children younger than one year was made in accordance with the Japanese pediatric guideline for the treatment and management of asthma. ${ }^{31}$ The efficacy of pranlukast dry syrup was evaluated by a parent or caregiver for asthma symptoms (attacks, cough) and an asthma diary. A prospective central registry of 192 cases collected from 93 medical institutions nationwide in Japan was used. No adverse drug reactions were observed among the 184 patients in the safety analysis. Of the 173 patients included in the efficacy analysis, 159 (91.9\%) showed improvement. Analysis of patient background factors showed no special concerns regarding drug efficacy. The frequency 
of wheezing and mild to moderate asthma attacks decreased significantly from week 4 to week 24 .

This prospective open-label study was not performed in a randomized or controlled fashion, primarily because of safety considerations in children. Current issues in relation to the treatment of patients with pediatric asthma include the efficiency of inhaled agents, theophylline clearance, and the paucity of approved medications. In addition, making a correct diagnosis of asthma is difficult in infancy. The available data suggest that pranlukast dry syrup is a useful drug for the treatment of asthma in infants aged younger than one year when used at the appropriate dose to weight ratio. However, a large-scale randomized controlled study will be needed to confirm the efficacy and safety of pranlukast dry syrup in infants, with appropriate diagnostic guidelines and monitoring by physicians who are familiar with pediatric asthma.

\section{Follow-up study of long-term use in children aged younger than one year}

A post-marketing surveillance study examined the safety and efficacy of long-term administration of pranlukast dry syrup in an actual clinical setting where children aged younger than one year and eligible for the previous prospective postmarketing survey could be followed up. ${ }^{32}$ The diagnosis of asthma was made in accordance with the Japanese pediatric guideline for the treatment and management of asthma. ${ }^{31}$ The efficacy of pranlukast dry syrup was evaluated by a parent or caregiver for asthma symptoms (attacks, cough) and an asthma diary.

Fifty cases from 33 medical institutions nationwide in Japan were considered. Of these, 34 continued medication for over one year. Only one adverse drug reaction (nonserious liver dysfunction) was reported in the 55 patients included in the safety analysis population, giving an incidence of $1.82 \%$. In this case, the increase in aspartate and alanine aminotransferase levels was considered to be moderate and resolved within 23 days of discontinuation of pranlukast dry syrup. However, because pranlukast is mainly metabolized by cytochrome P450 3A4, caution is required when it is coadministered with drugs that are predominantly metabolized by or inhibit the activity of cytochrome P450 3A4 enzymes. ${ }^{33}$ Among the 50 patients in the efficacy analysis population, $46(92.0 \%)$ showed improvement. Analysis of patient background factors showed no need for special concern regarding drug safety.

Thus far, the long-term efficacy and safety of pranlukast dry syrup has only been suggested by the results of this pro- spective open-label study, because a randomized controlled study is not feasible in infants for safety reasons. As described earlier, treatment of this patient population raises concerns regarding the efficacy of inhaled medication, theophylline clearance, and the few medications available. Pranlukast dry syrup appears to be useful for long-term treatment of bronchial asthma in pediatric patients younger than one your, particularly when used at the correct dose to weight ratio. However, further studies, especially randomized controlled trials in a larger patient population, are needed to verify the long-term efficacy and safety of this drug. Such studies would require appropriate guidelines for diagnosis, as well as monitoring by physicians who are respiratory specialists.

\section{Survey of quality of life}

A quality of life questionnaire (revised version 2001) designed for children with bronchial asthma and their parents or caregivers, and consisting of 15 questions for patients aged younger than 4 years and 20 questions for those aged 4 years and older (weighted at five points each) was used to evaluate the effect of pranlukast dry syrup on health-related quality of life in 56 pediatric patients with asthma. ${ }^{34}$ During this 4-week, open-label trial, pranlukast dry syrup improved overall health-related quality of life and physical and emotional domain scores in the children aged younger than 4 years $(n=20$, mean age 2.0 years $)$ and in those aged 4 years and older $(n=36$, mean age 6.6 years $)$ with mild to moderate asthma. Compared with baseline, overall health-related quality of life scores measured on a 100-point scale improved by $8-9$ points in patients aged younger than 4 years (from 54 to 62 points, $P<0.004$ ) and patients aged 4 years and older (from 80 to 89 points, $P<0.0001)$. Pranlukast dry syrup was confirmed to be useful as an antiasthmatic drug from the standpoint of quality of life for pediatric patients with bronchial asthma and their parents or caregivers.

\section{Randomized controlled trial of exercise-induced bronchospasm}

Pranlukast dry syrup inhibited exercise-induced bronchospasm in children with asthma in a randomized, multicenter, double-blind, placebo-controlled, two-period crossover trial. ${ }^{35}$ Patients were 17 children of mean age 11.0 (7-14) years with stable asthma and a history of typical symptoms of exercise-induced bronchospasm. Pranlukast dry syrup or a placebo was administered after dinner on the first day and after breakfast on the second day for each period of the study. All exercise activities were performed on a bicycle 
ergometer, and spirometric measurement of pulmonary function (forced vital capacity, forced expiratory volume in one second $\left[\mathrm{FEV}_{1}\right]$, peak expiratory flow, and expiratory flow at $50 \%$ and $25 \%$ of forced vital capacity) was collected before and after exercise. Compared with placebo, pranlukast dry syrup significantly inhibited any decrease in pulmonary function after exercise with regard to $\mathrm{FEV}_{1}$, maximal mid expiratory flow rate, peak expiratory flow, and expiratory flow at $50 \%$ of forced vital capacity at 15 and 30 minutes after exercise, and significantly decreased the area above the curve between 0 and 30 minutes and percent recovery. Pranlukast dry syrup also significantly decreased expiratory flow at $25 \%$ of forced vital capacity 30 minutes after exercise (Figure 2). Pranlukast dry syrup was confirmed to be safe and effective for management of asthma in pediatric patients with exercise-induced bronchospasm.

\section{Inhibition of aggravation by the common cold}

Viral infection is an important risk factor for aggravation of childhood asthma. ${ }^{36-40}$ Another recent study has investigated the ability of drugs to reduce aggravation of asthma triggered by the common cold. ${ }^{41}$ Asthma control was examined in 1014 Japanese pediatric patients with bronchial asthma. Occurrence of the common cold, asthma control, and drugs used for asthma control were evaluated using a modified Childhood Asthma Control Test in patients aged younger than 4 years and 4-11 years of age and the Asthma Control Test for patients aged $12-15$ years. ${ }^{42}$ No difference in asthma control was observed between the two age groups. The common cold and aggravation of asthma were significantly more prevalent in patients aged younger than 4 years. Control of asthma following aggravation by the common cold was

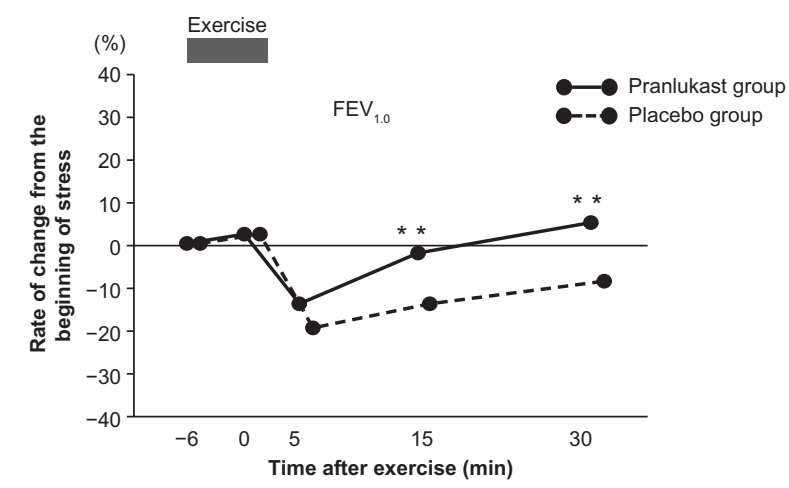

Figure 2 Change in forced expiratory volume in one second for pranlukast and placebo arms before and after exercise stress testing.

Notes: Unpaired t-test (pranlukast group vs placebo group). ${ }^{* * P}<0.01$; $\mathrm{FEV}_{1.0}$, forced expiratory volume in Ist second. significantly less effective in patients aged younger than 4 years compared with those aged 4 years and older. In the former group, asthma control was significantly more effective in those treated with leukotriene receptor antagonists. Asthma control did not differ between patients taking or not taking inhaled corticosteroids or long-acting $\beta 2$ stimulants. These findings indicate a high prevalence of the common cold in younger patients with childhood asthma and that the common cold aggravates asthma. Leukotriene receptor antagonists are useful for long-term asthma control in very young patients who suffer asthma attacks triggered by the common cold (Figure 3). Pranlukast dry syrup is a leukotriene receptor antagonist which is safe and effective for pediatric patients with bronchial asthma who develop asthma attacks due to the common cold.

\section{Peripheral eosinophil counts and serum IgE levels in children with food allergy}

Although the efficacy of the leukotriene receptor antagonists has already been established in bronchial asthma, their effect on food allergies remains unclear. A retrospective study examined 65 children of mean age $14 \pm 9.6$ (3-36) months with food allergies from 2005 to $2008 .{ }^{43}$ Of these, 32 patients served as a dietary control group by avoiding antigenic foods to which they had previously had an adverse reaction. The remaining 33 patients were treated with pranlukast dry syrup $7 \mathrm{mg} / \mathrm{kg}$ /day in addition to

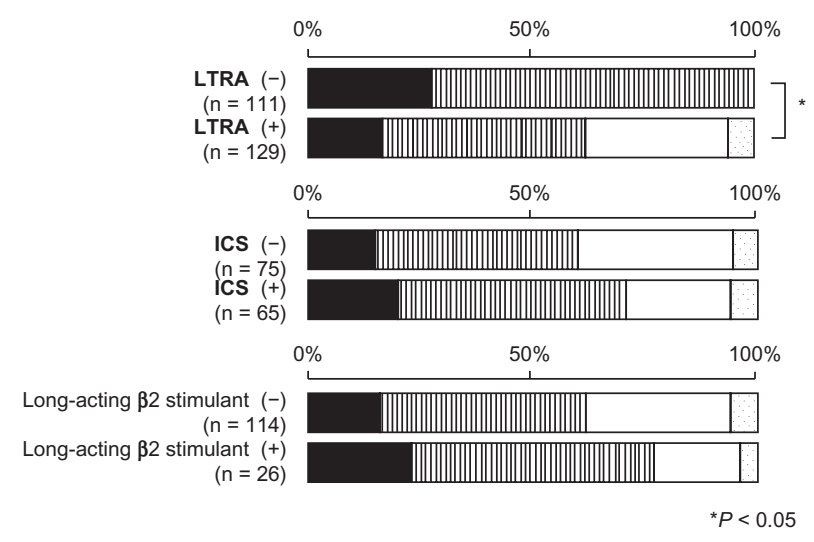

Figure 3 Effects of drugs on asthma control in patients younger than 4 years with a common cold. The black, striped, white, and dotted bars show patients who were uncontrolled, partly controlled, controlled, and not determined, respectively. Asthma control was evaluated using a modified Childhood Asthma Control Test filled out by the patient's parents. On the modified questionnaire, 26 or 27 points indicated asthma that was controlled, $20-25$ points indicated partially controlled asthma, and $<20$ points indicated uncontrolled asthma

Notes: *In this age group, control of asthma was significantly better for patients treated with LTRAs compared with those who did not receive LTRAs $(P<0.05)$. Abbreviations: ICS, inhaled corticosteroids; LTRA, leukotriene receptor antagonists. 
controlling their diet. Clinical symptoms and laboratory data before and after one year of treatment were compared between the two groups. Allergic symptoms improved in both the diet-controlled and pranlukast dry syrup groups, with no significant difference observed in clinical parameters between the groups after the one-year trial. Peripheral eosinophil count, serum IgE, interleukin (IL)-4, IL-5, IL-6, and eosinophil cationic protein levels in children with food allergies exceeded standard values in both groups. Although both the diet-controlled and pranlukast dry syrup groups showed decreased eosinophil counts $(-273 \pm 232$ per $\mu \mathrm{L}$ versus $-595 \pm 295$ per $\mu \mathrm{L}, P<0.05$ and $P<0.001$, respectively), only those treated with pranlukast dry syrup showed significantly decreased serum $\operatorname{IgE}(-73.5 \pm 115$ $\mathrm{IU} / \mathrm{mL}, P<0.01)$. Conversely, the control group showed significantly increased serum $\operatorname{IgE}(+159 \pm 138 \mathrm{IU} / \mathrm{mL}$, $P<0.01)$. The pranlukast dry syrup group also showed significantly decreased serum IL-4 $(54.5 \pm 31.0 \mathrm{pg} / \mathrm{mL}$ to $27.3 \pm$ $10.1 \mathrm{pg} / \mathrm{mL}), \operatorname{IL}-5(6.7 \pm 5.2 \mathrm{pg} / \mathrm{mL}$ to $5.0 \pm 0.4 \mathrm{pg} / \mathrm{mL})$, and eosinophil cationic protein $(45.4 \pm 15.0 \mu \mathrm{g} / \mathrm{L}$ to $15.0 \pm$ $9.8 \mu \mathrm{g} / \mathrm{L}, P<0.05$ each). Early intervention with pranlukast dry syrup may thus be effective in regulating eosinophil count and serum IgE, IL-4, IL-5, and eosinophil cationic protein levels.

These results support the potential efficacy of pranlukast dry syrup in young children with food allergies for preventing development of further allergies. As a result, pranlukast dry syrup is useful for pediatric patients with bronchial asthma and food allergies and has greater efficacy when used in combination with dietary control.

\section{Japanese cedar pollinosis}

A randomized, double-blind, placebo-controlled, crossover study using a artificial allergen exposure device (ie, an $\mathrm{OHIO}$ chamber $)^{44,45}$ investigated the efficacy and safety of pranlukast dry syrup in children aged 10-15 years with seasonal allergic rhinitis due to Japanese cedar pollen ${ }^{46}$ Eighty-four patients were enrolled and randomized in the treatment arm and 74 patients were included in the per protocol set. Patients were treated with either pranlukast dry syrup or placebo for one week, and were then exposed to Japanese cedar pollen in the OHIO chamber for 3 hours. Nasal symptom scores were recorded every 30 minutes during exposure. Pranlukast dry syrup significantly suppressed the change in total nasal symptom score change from baseline seen with placebo. The difference in least square averages of total nasal symptom scores between the group treated with pranlukast dry syrup and the placebo group was -0.37 (95\% confidence interval
$-0.54,-0.20, P<0.0001)$, confirming that pranlukast dry syrup significantly suppressed nasal symptoms, in particular sneezing, nasal discharge, and nasal obstruction. The greatest effect of pranlukast dry syrup was on nasal obstruction, with a significant improvement compared with placebo seen within 60 minutes of exposure. Pranlukast dry syrup is effective and safe for treating children with seasonal allergic rhinitis. As confirmed by the above results, pranlukast dry syrup is useful in view of the "one airway, one disease" concept, since pranlukast dry syrup is effective and safe in the treatment of bronchial asthma and allergic rhinitis in pediatric patients.

\section{Conclusion}

Asthma is one of the most common respiratory disorders seen in clinical practice, affecting up to $13 \%$ of the world's population. Inflammation is the predominant characteristic of asthma, and inhaled corticosteroids are recommended as first-line agents for control of the disease in patients of all ages. However, treatment with corticosteroids is often unable to control symptoms and progression of asthma fully. Leukotrienes have recently gained attention in research because they play a pivotal role in the airway inflammatory process, and specific drugs have been developed targeting them. Cysteinyl leukotriene antagonists have emerged as important therapeutic options, and show large potential clinical utility.

Pranlukast is approved for clinical use, including in the pediatric age group. Although adverse effects, such as headache, abdominal pain, rash, angioedema, pulmonary eosinophilia, and arthralgia have been reported, pranlukast is usually well tolerated and shows many positive effects in asthmatic patients. Current Global Initiative for Asthma guidelines recommend leukotriene receptor antagonists as second-line agents for patients with mild persistent asthma, and as addon therapy for reducing the dose of inhaled corticosteroids in patients with moderate or severe asthma, due to the complementary mechanisms of action of these agents. Leukotriene receptor antagonists may be particularly appropriate in a variety of clinical situations, including concomitant rhinitis, viral-induced wheeze, and exercise-induced bronchoconstriction, and in children aged younger than one year to reduce the frequency of asthma exacerbations.

CysLTs mimics many features of asthma, including bronchoconstriction, mucus secretion, microvascular leakage followed by edema, and eosinophil infiltration. CysLTs are also active in the proliferation of airway smooth muscle and collagen deposition underlying airway wall remodeling. 
Pranlukast prevents airflow obstruction, hyper-responsiveness, and inflammation associated with airway remodeling through antagonism of CysLTs.

Cysteinyl leukotrienes have been shown to have a potent pathophysiological effect in the upper and lower airways, suggesting that they are critical elements in the development, progression, and chronicity of the inflammatory response associated with various clinical presentations of asthma and allergic rhinitis.

These results indicate that pranlukast has great therapeutic potential in the treatment of asthma and allergic rhinitis. The pharmacological properties of pranlukast suggest that cysLT1 receptor antagonists are useful and beneficial as a new class of antiallergic and anti-inflammatory agents in the treatment and management of asthma and allergic rhinitis.

\section{Disclosure}

The author reports no conflicts of interest in this work.

\section{References}

1. National Institutes of Health. Global Initiative For Asthma: global strategy for asthma management and prevention. Available from: http:// www.ginasthma.org. Accessed October 29, 2012.

2. Weersink EJ, Postma DS, Aalbers R, de Monchy JG. Early and late asthmatic reaction after allergen challenge. Respir Med. 1994;88: 103-114.

3. O'Byrne PM. Leukotriene bronchoconstriction induced by allergen and exercise. Am J Respir Crit Care Med. 2000;161:S68-S72.

4. Kumlin M. Measurement of leukotrienes in humans. Am J Respir Crit Care Med. 2000;161:S102-S106.

5. Feldberg W, Kellaway CH. Liberation of histamine and formation of lysocithin-like substances by cobra venom. J Physiol. 1938;94: 187-226.

6. Kellaway $\mathrm{CH}$, Trethewie ER. The liberation of a slow reacting smoothmuscle-stimulating substance in anaphylaxis. Q J Exp Physiol. 1940;30: 121-145.

7. Brocklehurst WE. The release of histamine and formation of a slowreacting substance (SRS-A) during anaphylactic shock. $J$ Physiol. 1960;151:416-435.

8. Samuelsson B. The discovery of the leukotrienes. Am J Respir Crit Care Med. 2000;161:52-56.

9. Dunn CJ, Goa KL. Zafirlukast: an update of its pharmacology and therapeutic efficacy in asthma. Drugs. 2001;61:285-315.

10. Murphy RC, Hammarstrom S, Samuelsson B. Leukotriene C: a slow-reacting substance from murine mastocytoma cells. Proc Natl Acad Sci U S A. 1979;76:4275-4279.

11. Samuelsson B. Leukotrienes: mediators of immediate hypersensitivity reactions and inflammation. Science. 1983;220:568-575.

12. Lewis RA, Austen KF, Soberman RJ. Leukotrienes and other products of the 5-1ipoxygenase pathway: biochemistry and relation to pathobiology in human diseases. $N$ Engl J Med. 1990;323: 645-655.

13. Lynch KR, O'Neill GP, Liu Q, et al. Characterization of the human cysteinyl leukotriene CysLT 1 receptor. Nature. 1999;399: 789-793.

14. Sarau HM, Ames RS, Chambers J, et al. Identification, molecular cloning, expression, and characterization of a cysteinyl leukotriene receptor. Mol Pharmacol. 1999;56:657-663.
15. Nothacker H-P, Wang Z, Zhu Y, et al. Molecular cloning and characterization of a second human cysteinyl leukotriene receptor: discovery of a subtype selective agonist. Mol Pharmacol. 2000;58: 1601-1608.

16. Heise CE, O'Dowd BF, Figueroa DJ, et al. Characterization of the human cysteinyl leukotriene 2 receptor. J Biol Chem. 2000;275: 30531-30536.

17. Leff AR. Discovery of leukotrienes and development of antileukotriene agents. Ann Allergy Asthma Immunol. 2001;86 Suppl:4-8.

18. O'Byrne PM. Leukotrienes in the pathogenesis of asthma. Chest. 1997;111 Suppl:27S-34S.

19. Dahlen B, Dahlen SE. Leukotrienes as mediators of airway obstruction and inflammation in asthma. Clin Exp Allergy. 1995;25 Suppl 2: $50-54$.

20. Dahlen SE. Leukotriene receptors. Clin Rev Allergy Immunol. 1999;17:179-191.

21. Dahlen SE. Pharmacological characterization of leukotriene receptors. Am J Respir Crit Care Med. 2000;161:S41-S45.

22. Labat C, Ortiz JL, Norel X, et al. A second cysteinyl leukotriene receptor in human lung. J Pharmacol Exp Ther. 1992;263:800-805.

23. Walch L, Norel X, Back M, et al. Pharmacological evidence for a novel cysteinyl-leukotriene receptor subtype in human pulmonary artery smooth muscle. Br J Pharmacol. 2002;137:1339-1345.

24. Yonetomi Y, Fujita M, Nakagawa N, et al. Preclinical pharmacology of pranlukast. Clin Exp Allergy Rev. 2001;1:210-217.

25. Mikawa H, Baba M. Efficacy and safety of a leukotriene antagonist; ONO-1078 dry syrup, on pediatric bronchial asthma - dose finding study in a multi-center, open trial. Rinsho Iyaku. 1997;13(2):395-421. Japanese.

26. Mikawa $H$, Baba M, Nakashima M. Clinical usefulness of a leukotriene antagonist; pranlukast dry syrup on pediatric bronchial asthma in multi-center comparative double-blind clinical study with oxatomide dry syrup. Rinsho Iyaku. 1997;13:423-456. Japanese.

27. Iikura $Y$, Saito H, Sakaguchi N, et al. Efficacy and safety of long-term use of a leukotriene $\mathrm{C}_{4}, \mathrm{D}_{4}$ and $\mathrm{E}_{4}$ antagonist, ONO-1078 dry syrup, in pediatric bronchial asthma. Rinsho Iyaku. 1997;13:1015-1039. Japanese.

28. Toyoshima K, Kameda M, Mikawa H, et al. Efficacy and safety of a long-term (12 weeks) use of a leukotriene $\mathrm{C}_{4}, \mathrm{D}_{4}$ and $\mathrm{E}_{4}$ antagonist, ONO-1078 dry syrup, in asthmatic children. Rinsho Iyaku. 1997;13: 1041-1071. Japanese.

29. Hata D, Shiota M, Tamura Y, et al. The effectiveness of long-term treatment with pranlukast in pediatric patients with mild to moderate asthma. Arerugi. 2002;51:400-410. Japanese.

30. Iwata T, Kurihara K, Odajima Y, et al. Safety and effectiveness of Onon dry syrup $10 \%$ (pranlukast hydrate) in children aged less than 1 year with bronchial asthma - prospective study. Jpn J Pediatr Allergy Clin Immunol. 2009;23:629-642. Japanese.

31. Nishimuta T, Kondo N, Hamasaki Y, et al. Japanese guideline for childhood asthma. Allergol Int. 2011;60:147-169.

32. Iwata T, Kurihara K, Odajima Y, et al. Safety and effectiveness of Onon dry syrup (pranlukast hydrate) in children aged less than 1 year with bronchial asthma - follow-up study of long-term use. Jpn J Pediatr Allergy Clin Immunol. 2010;24:693-704. Japanese.

33. Inoue R, Teramoto T, Nakade S, et al. Population pharmacokinetics of pranlukast hydrate dry syrup in children with bronchial asthma. Allergol Int. 2003;52:213-218.

34. Kondo N, Teramoto T, Inoue R, et al. Evaluation before and after pranlukast administration with the QOL questionnaire (revised version 2001) for pediatric patients with bronchial asthma and their parents or caregivers. Arerugi. 2002;51:421-429. Japanese.

35. Nishima S, Furusho K, Morikawa A, et al. Pranlukast inhibits exerciseinduced bronchospasm in asthmatic children: a randomized, multicenter, double-blind, placebo-controlled two-period crossover trial. Pediatr Asthma Allergy Immunol. 2005;18:5-11.

36. Sigurs N, Bjarnason R, Sigurbergsson F, Kjellman B. Respiratory syncytial virus bronchiolitis in infancy is an important risk factor for asthma and allergy at age 7. Am J Respir Crit Care Med. 2000;161:1501-1507. 
37. Jackson DJ, Gangnon RE, Evans MD, et al. Wheezing rhinovirus illnesses in early life predict asthma development in high-risk children. Am J Respir Crit Care Med. 2008;178:667-672.

38. Van Den Hoogen BG, DeJong JC, Groen J, et al. A newly discovered human pneumovirus isolated from young children with respiratory tract disease. Nat Med. 2001;7:719-724.

39. Yoshihara S, Yamada Y, Abe T, Linden A, Arisaka O. Association of epithelial damage and signs of neutrophil mobilization in the airways during acute exacerbations of paediatric asthma. Clin Exp Immunol. 2006;144:212-216.

40. Laprise C, Laviolette M, Boutet M, Boulet LP. Asymptomatic airway hyperresponsiveness: relationships with airway inflammation and remodelling. Eur Respir J. 1999;14:63-73.

41. Yoshihara S, Fukuda H, Abe T, et al. Inhibition of common cold-induced aggravation of childhood asthma by leukotriene receptor antagonists. Allergol Int. 2012;61:405-410.
42. Schatz M, Sorkness CA, Li JT, et al. Asthma Control Test: reliability, validity, and responsiveness in patients not previously followed by asthma specialists. J Allergy Clin Immunol. 2006;117:549-556.

43. Yamakawa Y, Ohtsuka Y, Ohtani K, et al. Effects of leukotriene receptor antagonists on peripheral eosinophil counts and serum IgE levels in children with food allergy. Drugs R D. 2010;10:147-154.

44. Hashiguchi K, Tang H, Fujita T, et al. Validation study of the OHIO chamber in patients with Japanese cedar pollinosis. Int Arch Allergy Immunol. 2009;149:141-149.

45. Hashiguchi K, Tang H, Fujita T, et al. Preliminary study on Japanese cedar pollinosis in a artificial exposure chamber (OHIO chamber). Allergol Int. 2007;56:125-130.

46. Wakabayashi K, Hashiguchi K, Kanzaki S, et al. Pranlukast dry syrup inhibits symptoms of Japanese cedar pollinosis in children using OHIO Chamber. Allergy Asthma Proc. 2012;33:1-8.

\section{Publish your work in this journal}

Pediatric Health, Medicine and Therapeutics is an international, peerreviewed, open access journal publishing original research, reports, editorials, reviews and commentaries. All aspects of health maintenance, preventative measures and disease treatment interventions are addressed within the journal. Practitioners from all disciplines are invited to submit their work as well as healthcare researchers and patient support groups. The manuscript management system is completely online and includes a very quick and fair peer-review system. Visit http://www.dovepress.com/ testimonials.php to read real quotes from published authors.

Submit your manuscript here: http://www.dovepress.com/pediatric-health-medicine-and-therapeutics-journal 